\title{
Volitional rehabilitative assessments in patients admitted in a post-intensive care step down unit. A feasibility study
}

\author{
Mara Paneroni1, Francesco D’Abrosca², Georges Fokom1, Laura Comini³, Michele Vitacca1 \\ 1 Respiratory Rehabilitation Division, Maugeri Clinical Scientific Institutes, Lumezzane (BS) \\ 2 Department of Neurosciences, Unit of Neurology, IRCCS Ca'Granda Foundation Ospedale Maggiore \\ Policlinico, Milano \\ 3 Health Directorate, Maugeri Clinical Scientific Institutes, Lumezzane (BS), Italy
}

\begin{abstract}
A high variability in functional tests and activities used during the pulmonary rehabilitation has been observed in post-intensive care unit (ICU) patients, and the best battery of tests to adopt has not been described yet. We tested in patients admitted in a post-ICU Step Down Unit the ability to perform the more frequent functional volitional tests. The relations of each single volitional test with general disability and dyspnea at discharge were also evaluated. Ten volitional tests including: bedside spirometry test (ST: $\mathrm{FEV}_{1} \%$, FVC\%), maximal inspiratory pressure (MIP), maximal expiratory pressure (MEP),
\end{abstract}

Corresponding author: Mara Paneroni, Respiratory Rehabilitation Division, Istituti Clinici Maugeri IRCCS, Via G. Mazzini 129, 25065 Lumezzane (BS), Italy. Tel. +39.30 .8253122 - Fax: +39.30 .8253189 .

E-mail: mara.paneroni@icsmaugeri.it

Key words: Weaning; mechanical ventilation; assessment; physical therapy; disability.

Contributions: MP, study design, statistical analysis, manuscript drafting and critical revision for important intellectual content; MV, substantial contributions to the concept, manuscript drafting and critical revision for important intellectual content; LC, substantial contributions to the concept and the figures, manuscript critical revision for important intellectual content, final version approval; GF, FDA, data acquisition, manuscript critical revision for important intellectual content, final version approval. All Authors approved the final version to be published.

Conflict of interest: the Authors have no conflict of interest to disclose.

Acknowledgments: the Authors wish to thank Rosemary Allpress for the English revision of the manuscript.

Received for publication: 17 August 2016

Accepted for publication: 6 February 2017

CCopyright M. Paneroni et al., 2017

Tipografia PI-ME Editrice, Italy

Monaldi Archives for Chest Disease 2017; 87:764

doi: 10.4081/monaldi.2017.764

This article is distributed under the terms of the Creative Commons Attribution Noncommercial License (by-nc 4.0) which permits any noncommercial use, distribution, and reproduction in any medium, provided the original author(s) and source are credited.
Peak Expiratory Flow during Cough (PCEF), Quadriceps Muscle Strength (QMS), latissimus Dorsi and teres Major Strength (DMS), Brachial biceps Muscle Strength (BMS), effort tolerance measured by sit-to-stand test, Takahashi test and 6-Min Walking Test (6MWT), were evaluated in post-ICU patients at entry and discharge from inhospital rehabilitation. General disability was assessed by Barthel Index, while dyspnea by Borg scale.

At admission, $>70 \%$ of subjects performed muscle strength test, while $<25 \%$ performed respiratory and effort tolerance tests. At discharge, feasibility of spirometry, respiratory muscle strength and effort tolerance tests improved (all, $\mathrm{p}<0.001$ ); 6MWT was the least feasible. At discharge, cardiorespiratory patients were more capable to perform tests compared to neurological ones. All outcome measures, with exception of $\mathrm{FEV}_{1} \%$, and $\mathrm{FVC} \%$, were significantly related to the disability score.

Peripheral muscle exercises showed the highest feasibility, spirometry and leg effort tolerance the lowest. Motor disability was explained mainly by the peripheral muscle strength. The study of non-volitional outcome measures and tests linked to a protocol-driven intervention should be performed in this specific population.

\section{Introduction}

In the last 15 years, availability of beds in intensive care unit (ICU) and new technologies coupled with improved levels of care have highlighted a new category of subjects labeled "ICU survivors" in whom hospitalization and recovery may be abnormally prolonged [1]. Their physical disabilities include mainly peripheral and respiratory muscle wasting, weakness, neuromyopathies and poor nutritional status [2]. Post ICU-discharge care for this population often requires transfer to a Post-ICU Step Down Unit (P-ICU) [3,4]. Previous reports have shown that rehabilitation in this population is safe and feasible, involving respiratory muscle strength, limb and arm muscles strength, activities of daily living (ADL) functional scores and effort tolerance [5-8]. Two recent reviews by Elliot et al. [9] and Connolly et al. [10] describe a high variability in muscle strength evaluations, functional activity tests, walking assessments, and patient-centered outcomes such as healthrelated quality of life in survivors of ICU. The Authors conclude that sensitivity and validity of measures in survivors of a critical illness have not yet been established and new tools need to be developed to appropriately assess the weakness and poor physical function in order to measure effectiveness of interventions $[9,10]$. In fact, the majority of studies carried out take into consideration only some aspects of patient's disability, but no study has performed a comprehensive and objective rehabilitative assessment with the most common measures used in this field to detect their feasibility. 
To this aim, in patients who underwent P-ICU rehabilitation, we proposed a multidimensional assessment to evaluate: i) rate subjects' ability to perform each test at admission and discharge analyzing the difference according to diagnosis; and ii) the relationship of each single volitional test with general disability and dyspnea at discharge.

\section{Materials and Methods}

All study procedures were carried out in conformity with the Declaration of Helsinki. The institutional review board (Istituti Clinici Maugeri IRCCS, deliberation $\mathrm{N}^{\circ} 751$ ) approved the study. Written informed consent was obtained from all participants.

\section{Design}

This was a cohort prospective observational study.

\section{Participants}

From January 2010 to June 2011 we enrolled all consecutively discharged post-acute critical care patients who had: i) a recent $(\leq 3$ months) episode of acute respiratory failure (ARF); ii) an ICU stay of at least 20 consecutive days with difficulty weaning from mechanical ventilation (MV); iii) completed a P-ICU rehabilitation program. Following critical care, the P-ICU rehabilitation program consisted of clinical stabilization, weaning attempts from MV by progressively reducing inspiratory support or increasing time of spontaneous breathing, and individualized/integrated physiotherapy administered by a dedicated physiotherapist (PT) who provided an individually-tailored program of passive and active assisted exercises and mobilization, maintenance of body posture, electrical stimulation of leg muscles, sitting and standing postural exercises, limb strength/endurance training by cycle ergometer or treadmill, walking assistance, lung volume recruitment, and bronchial hygiene. The intervention was 1 hour/day which could be performed as a single or in two 30-min sessions 6 days per week. Besides possible need for MV, supplemental oxygen was administered to maintain oxy-hemoglobin saturation $\left(\mathrm{O}_{2}\right.$ sat) greater than $90 \%$ during exercise. The respiratory assistance was gradually reduced over time and the PT constantly monitored the patient for signs of cardiorespiratory distress during the sessions.

Patients were excluded if they: were under 18 years of age, had cardiovascular instability, amyotrophic lateral sclerosis, remained critically ill, had electrodiagnostic evidence of myopathy/polyneuropathy, had multi-organ failure, required hemodialysis, had a life expectancy less than 6 months, lived more than $80 \mathrm{~km}$ from the study center, were transferred to another hospital or nursing home, refused to participate, or were enrolled in other trials. The multidisciplinary rehabilitative program was based on a close collaboration between doctors, nurses and physiotherapists. The details of the program are described elsewhere [8].

\section{Outcomes measures}

All patients were tested in a P-ICU rehabilitation unit from the Istituti Clinici Maugeri IRCCS of Lumezzane (BS). At admission (T0), the following parameters were collected:

- Anthropometric data, e.g. age, gender, body mass index (BMI)

- APACHE II score 11

- Main diagnosis, based on which subjects were subdivided into two main groups (cardiorespiratory diseases and neurological diseases)

- Presence of tracheostomy

- MV use for weaning attempts
At (T0) and hospital discharge (T1), subjects underwent ten volitional tests, conducted by a PT consisted of:

i) a bedside spirometry test (ST) to measure the FEV1 and FVC with a portable spirometer (V Max, Sensormedics; Carefusion, Franklin Lakes, NJ, USA) [12];

ii) maximal inspiratory pressure (MIP);

iii) maximal expiratory pressure (MEP) according to the method of Black and Hyatt [13] with a portable differential pressure transducer (Honeywell 300 manometer; Freeport, IL, USA) using a flanged mouthpiece [14];

iv) peak cough expiratory flow (PCEF) with a peak flow meter (MiniWright, Clement Clarke International Ltd., Harlow, UK) using a flanged mouthpiece or an oro-nasal mask (Cristal, Koo Industries Co., Ltd., Shanghai, China) $[15,16]$;

v) quadriceps muscle strength (QMS);-

vi) brachial biceps muscle strength (BMS);

vii) latissimus dorsi and teres major muscle strength (DMS); All peripheral muscle function evaluations were carried out by a manual test using the Medical Research Council (MRC) scale to measure the range of muscle strength, with score ranging from 0 (no visible or palpable muscle contraction) to 5 (movement through the complete range of motion against gravity and maximum resistance) [17]. One leading muscle, possibly on the dominant limb, was tested: QMS was performed in sitting or supine position depending on the patient's clinical condition, as recommended by Hough et al. [18]. DMS and BMS were tested in lateral decubitus or in a sitting position as previously recommended [19];

viii) effort tolerance measured by the 30 -second sit-to-stand test performed using a 43-cm high chair without arm rests as described by Jones et al. [20]. Subjects were asked to rise from the chair and sit down as many times as possible without using the arms as a support. The number of completed repetitions in 30 seconds was recorded;

ix) effort tolerance measured by an incremental test for unsupported upper limbs as described by Takahashi et al. [21] was used. The total duration (seconds) of the test was recorded;

x) effort tolerance measured by the 6-minute walk test (6MWT) according to international guidelines [22]. The total distance (meters) reached was recorded.

Inability to perform a test (in which case 0 points were assigned) included:

1. low level of consciousness and cognitive functions (GCS <10) [23];

2. low compliance with instructions given for each test;

3. limitation in performing the assessments as follows: i) during spirometry tests, the patient did not generate flow detectable by the spirometer or was totally dependent $(\mathrm{H} 24)$ on mechanical ventilation; ii) during respiratory muscle strength tests, the patient was not able to sustain the effort for at least 1.5 seconds, or was unable to perform three maneuvers with less than $20 \%$ of difference between the values [13] or was totally dependent (24/24 h) on MV; iii) during PCEF, the patient was not able to stake retain air in the lung closing the glottis or was not able to produce at least three attempts with values differing less than $40 \mathrm{~L} / \mathrm{min}$ [24]; iv) the peripheral muscle strength value was 0 (no visible/palpable contraction); v) during 30 seconds sit-to-stand test, the patient was not able to reach and maintain the sitting position or needed arm aid or external help to reach the standing position; vi) during the Takahashi test, the patient was not able to reach and/or maintain the sitting position or was unable to perform the test correctly; vii) during the 6MWT, the patient was not able to walk alone or for more than 20 meters or was totally dependent (24/24 h) on mechanical ventilation. 
In addition, at $\mathrm{T} 0$ and $\mathrm{T} 1$, the general motor disability and dyspnea were recorded using the Barthel index (score ranging from $0=$ totally dependent to $20=$ totally independent) [25] and BORG scale $(0=$ no dyspnea, 10 = maximal dyspnea) [26].

\section{Data analysis}

Statistical analyses were performed using the software package STATA 12.1 (StataCorp LP, USA). All variables were expressed as mean and standard deviation (SD) or as a percentage. The change obtained by the patients able to execute the rehabilitation tests at admission and discharge was analyzed using chi-square test.

Univariate linear regression analysis was performed on data at discharge to show relation between volitional tests and Barthel Index or Dyspnea Score (Borg score). For all statistical tests a p-value $<0.05$ was considered as statistically significant.

\section{Results}

From January 2010 to June 2011, 67 subjects were enrolled as described in Figure 1. Their main clinical characteristics were shown in Table 1.

Subjects were middle aged, half of them presented neurological diseases (stroke 52\%, post cardiac arrest 15\%, Guillain-Barré syndrome $7 \%$, non-degenerative neuromuscular diseases 18\%, Parkinson's dis- ease 4\%, and astrocytoma 4\%); they had an ICU stay longer than 30 days; most required tracheostomy (72\%) or MV use $(60 \%)$, while clinical conditions were stable.

Figure 2 describes the overall rate of feasibility (panel A), i.e. "performability", of each rehabilitative volitional test administered at admission and discharge and the rate of feasibility in cardiorespiratory and neurological patients (panel B and C, respectively). At admission, only peripheral muscle evaluations showed a good overall feasibility (70\%), i.e. they could be performed by most subjects, while few subjects (less than 25\%) could perform the respiratory and effort tolerance tests. In particular, no one could perform the 6MWT. At discharge, the overall feasibility of respiratory function, respiratory muscle tests and effort tolerance tests improved significantly $(\mathrm{p}<0.001)$. At discharge, all peripheral muscle tests were highly feasible; respiratory muscle and arm effort tolerance tests could be carried out by more than $50 \%$ of subjects, while the spirometry test, PCEF and sit-to-stand test were slightly performable. The 6MWT showed the least overall feasibility, i.e. only $25 \%$ of subjects at discharge were able to perform it.

Concerning differences between cardiorespiratory and neurological patients, at the admission the only volitional tests significantly different $(\mathrm{p}<0.001)$ in performability were QMS, BMS and DMS that were higher in cardiorespiratory patients compared to neurological ones. On the contrary, at the discharge cardiorespiratory patients were significantly more able to perform all the measures compared to neurological ones $(\mathrm{p}<0.05)$ (Table 2, Figure 2).

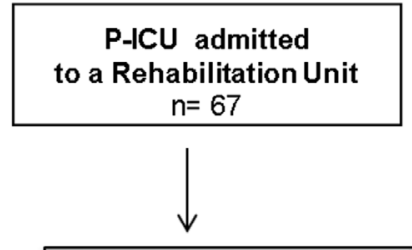

TO in-hospital program

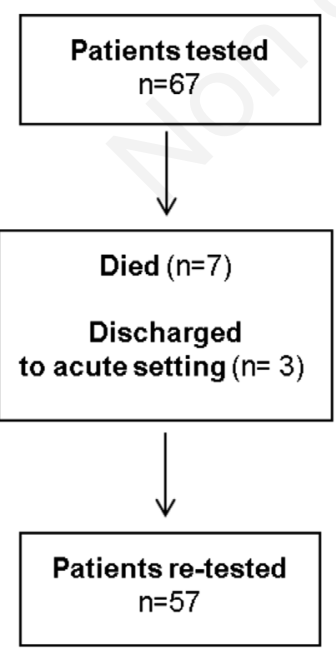

\section{Type of exercises}

(i) a bedside spirometry test (ST)

(ii) maximal inspiratory pressure (MIP)

(iii) maximal expiratory pressure (MEP)

(iv) peak cough expiratory flow (PCEF)

(v) quadriceps muscle strength (QMS)

(vi) brachial biceps muscle strength (BMS)

(vii) latissimus dorsi and teres major muscle strength (DMS)

(viii) sit-to-stand test

(ix) unsupported upper limbs (Takahashi test)

(x) 6-minute walk test (6MWT)

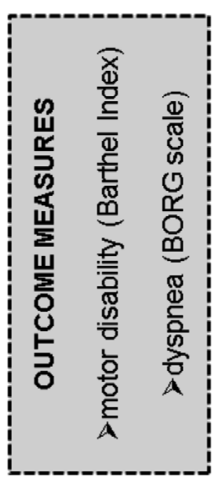

T1 in-hospital retest

Figure 1. Flow chart of the study. 
Table 1. Characteristics of the studied subjects at admission to our rehabilitation unit.

\begin{tabular}{lc} 
Characteristics & \\
Enrolled subjects, n & 67 \\
Age (years), mean (SD) & $66(12)$ \\
Males, \% & 52 \\
BMI, kg/m², mean (SD) & $26(5)$ \\
Diagnosis & \\
$\quad$ Neurological diseases n, \% & $27(40)$ \\
$\quad$ Cardiorespiratory diseases n, \% & $40(60)$ \\
$\quad$ Post cardiac surgery n, \% & $13(19)$ \\
$\quad$ ARF n, \% & $12(18)$ \\
$\quad$ COPD n, \% & $15(22)$ \\
ICU LOS, days, mean (SD) & $36(39)$ \\
Rehabilitation unit LOS, days, mean (SD) & $86(66)$ \\
pH, mean (SD) & $7.44(0.07)$ \\
PaO 2 FiO & , mean (SD) \\
PaCO & $253(84)$ \\
Apache II score, mean (SD) $_{\text {Tracheostomized, n (\%) }}$ & $51(14)$ \\
Ventilated, n (\%) & $12.0(5.7)$ \\
\hline
\end{tabular}

BMI: Body Max Index; ARF: Acute Respiratory Failure, $\mathrm{FiO}_{2}$ : Inspiratory rate of oxygen, LOS: length of stay; $\mathrm{PaO}_{2}$ : partial pressure of oxygen; $\mathrm{PaCO}_{2}$ : partial pressure of carbon dioxide.

Subjects performed 240 (SD 196) rehabilitation sessions during the 86 (SD 66) days of stay in the rehabilitation facility. In-hospital mortality was observed in 7 subjects (10\%) and the drop-out rate (due to 3 subjects' transfer to another hospital) was $4 \%$.

Forty percent of subjects had ventilated through tracheostomy at T0 and $19 \%$ still required MV at T1. Table 2 describes value of volitional measures in survived patients that were able to carry out volitional tests.

Table 3 reports univariate regression models between the Barthel Index and all the volitional tests evaluated at discharge. All outcome measures with the exception of spirometry (FEV1\%, and FVC\%) were significantly related to the disability score. As described by the regression coefficient (Table 3), peripheral muscle tests were the measures with the highest added effect on disability. Only spirometry (FEV1\%, and FVC\%) and cough ability (PCEF) were significantly related to the Dyspnea Borg Score in the univariate regression model (e.g., dyspnea versus all the volitional tests), but their impact on dependent variable is probably clinically not so relevant as evidenced by the very low power of the model (FEV1\% regression coefficient $0.036, \mathrm{R}^{2}$ 0.278, $\mathrm{p}=0.009, \mathrm{FVC} \%$ regression coefficient $-0.036, \mathrm{R}^{2}$ $0.198, \mathrm{p}=0.029$, PCEF regression coefficient $-0,012, \mathrm{R}^{2} 0.381$, $\mathrm{p}=0.002 ;$ Table 4 ).

\section{Discussion}

In a sample of subjects with a recent ICU stay and discharged from a rehabilitation facility, the present study has shown that: i) among different volitional tests, peripheral muscle evaluations result the most feasible, while spirometry and leg effort tolerance were the least feasible; ii) at discharge, cardiorespiratory patients were generally more able to perform tests compared to neurological patients; iii) motor disability is explained mainly by peripheral muscle strength; iv) dyspnea is explained mainly by respiratory function tests.

\section{A Whole population}

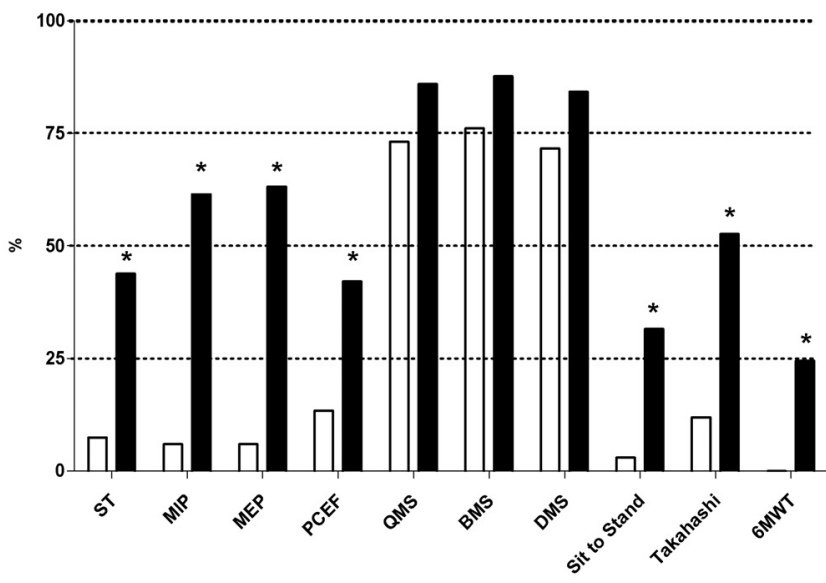

\section{B Cardiorespiratory Patients * * *}

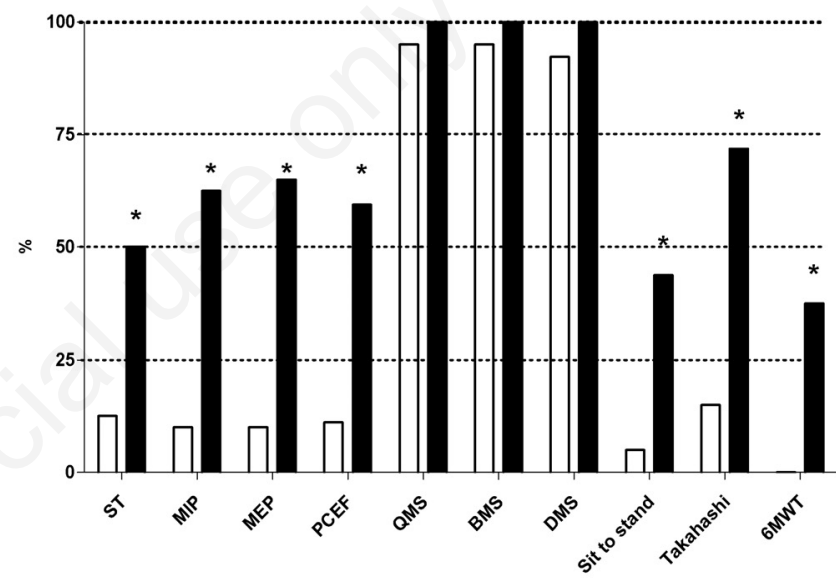

\section{Neurological Patients}

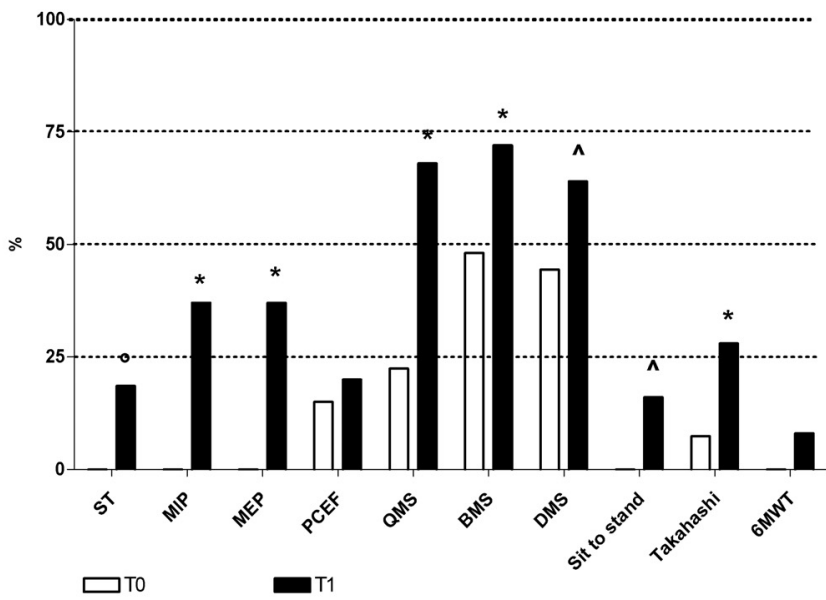

Figure 2. Percentage of subjects defined as able to perform each test (for details see methods) at admission (T0, white bars) and at discharge (T1, black bars) of P-ICU unit. ${ }^{\wedge} \mathrm{p}<0.05 ;{ }^{\circ} \mathrm{p}<0.02 ;{ }^{*} \mathrm{p}<0.001$ vs T0. ST: spirometry test; MIP: maximal inspiratory pressure; MEP: maximal expiratory pressure; PCEF: peak cough expiratory flow; QMS: quadriceps muscle strength; BMS: biceps muscle strength; DMS: latissimus dorsi and teres major muscle strength; 6MWT: six-minute walking test. 
Table 2. Value of volitional measures in patients who were able to perform the tests.

\begin{tabular}{|c|c|c|c|c|c|c|c|c|c|c|c|c|}
\hline & \multicolumn{4}{|c|}{ Overall } & \multicolumn{4}{|c|}{ Cardiorespiratory patients } & \multicolumn{4}{|c|}{ Neurological patients } \\
\hline & T0 & & T1 & & T0 & & T1 & & T0 & & T1 & \\
\hline & Mean (sd) & n & Mean (sd) & $\mathbf{n}$ & Mean (sd) & $\mathbf{n}$ & Mean (sd) & $\mathbf{n}$ & Mean (sd) & $\mathbf{n}$ & Mean (sd) & $\mathbf{n}$ \\
\hline FEV1, \% & $32.2(23.6)$ & 5 & $59.6(29.0)$ & 25 & $32.2(23.6)$ & 5 & $58.8(29.1)$ & 20 & ----- & 0 & $62.8(31.5)$ & 5 \\
\hline FVC, $\%$ & $58.4(21.1)$ & 5 & $69(24.6)$ & 25 & $58.4(21.1)$ & 5 & $68.8(24.4)$ & 20 & 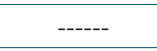 & 0 & $70.0(27.9)$ & 5 \\
\hline MIP, $\mathrm{cmH}_{2} \mathrm{O}$ & $37(10.1)$ & 4 & $44.9(20.2)$ & 35 & $37(10.1)$ & 4 & $51.5(19.1)$ & 25 & ------ & 0 & $28.6(12.3)$ & 10 \\
\hline MEP, $\mathrm{cmH}_{2} \mathrm{O}$ & $59.3(17.7)$ & 4 & $54.1(29.4)$ & 36 & $59.3(17.7)$ & 4 & $58.23(27.43)$ & 26 & 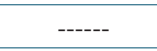 & 0 & $43.2(33.2)$ & 10 \\
\hline PCEF, L/min & $120.0(36.7)$ & 9 & $205.8(120.7)$ & 28 & $138.3(23.2)$ & 6 & $217.4(100.2)$ & 24 & $83.3(32.1)$ & 3 & $162.0(111.2)$ & 4 \\
\hline QMS, Kg & $2.92(0.79)$ & 49 & $3.88(0.89)$ & 58 & $2.86(0.81)$ & 38 & $4.15(0.54)$ & 40 & $3.18(0.68)$ & 11 & $3.34(1.19)$ & 18 \\
\hline BMS, Kg & $3.12(0.87)$ & 51 & $3.99(0.96)$ & 59 & $3.15(0.83)$ & 38 & $4.36(0.56)$ & 40 & $3.01(0.97)$ & 13 & $3.33(1.16)$ & 19 \\
\hline DMS, Kg & $2.77(1.04)$ & 49 & $3.88(0.89)$ & 56 & $2.17(1.05)$ & 37 & $4.17(0.66)$ & 40 & $2.97(1.01)$ & 12 & $3.32(1.40)$ & 16 \\
\hline Sit-to -stand, repetitions & $15.5(7.8)$ & 2 & $13.28(7.7)$ & 21 & $15.5(7.8)$ & 2 & $13.3(7.7)$ & 18 & ----- & 0 & $11.8(9.2)$ & 3 \\
\hline Takahashi, seconds & $119(58)$ & 8 & $181(99)$ & 35 & $129(62)$ & 6 & $176(105)$ & 29 & $88(46)$ & 2 & $200(81)$ & 6 \\
\hline 6MWT, meters & ----- & 0 & $294(92)$ & 17 & ------ & 0 & $278(70)$ & 15 & ----- & 0 & 390 (184) & 2 \\
\hline
\end{tabular}

ST: spirometry test; MIP: maximal inspiratory pressure; MEP: maximal expiratory pressure; PCEF: peak cough expiratory flow; QMS: quadriceps muscle strength; BMS: biceps muscle strength; DMS: latissimus dorsi and teres major muscle strength; 6MWT: six-minute walking test; T0: at admission; T1: at discharge; \%: percentage of predicted value; $\mathrm{cmH}_{2} \mathrm{O}$ : centimeters of water; L: liters; Min: minutes; n: number of patients who were able to perform the volitional measure; ------: patients unable to execute the tests.

Table 3. Univariate regression model of Barthel Index as dependent variable: relationship with each volitional test evaluated at discharge.

\begin{tabular}{lcccc} 
Models & Slope & $\mathrm{t}$ & $\begin{array}{c}\text { P-value } \\
\text { coefficient }\end{array}$ & $\mathrm{R}^{2}$ \\
FEV 1 \% pred & 0.06 & 1.38 & 0.18 & 0.11 \\
\hline FVC \% pred & 0.05 & 0.74 & 0.06 & 0.47 \\
\hline MIP \% pred & 0.19 & 4.3 & $<0.002$ & 0.29 \\
\hline MEP \% pred & 0.12 & 2.9 & 0.006 & 0.27 \\
\hline PCEF, L/min & 0.02 & 2.1 & 0.047 & 0.17 \\
\hline QMS score & 3.72 & 14.8 & $<0.001$ & 0.65 \\
\hline BMS score & 3.69 & 12.1 & $<0.001$ & 0.60 \\
\hline DMS score & 3.58 & 14.0 & $<0.001$ & 0.63 \\
\hline Sit-to-stand, n of repetitions & 0.68 & 7.6 & $<0.001$ & 0.46 \\
\hline Takahashi, seconds & 0.05 & 9.0 & $<0.001$ & 0.59 \\
\hline 6MWT, meters & 0.04 & 8.3 & $<0.001$ & 0.41 \\
\hline $\begin{array}{l}\text { MIP: maximal inspiratory pressure; MEP: maximal expiratory pressure; PCEF: peak cough expiratory } \\
\text { flow; QMS: quadriceps muscle strength; BMS: biceps muscle strength; DMS: latissimus dorsi and teres } \\
\text { major muscle strength; 6MWT: six-minute walking test. }\end{array}$ & &
\end{tabular}

Critical illness and treatment may cause a decline in subjects' functional capacity, which affects the recovery trajectory for ICU survivors [27]. Despite that, in the rehabilitative approach to post-critically ill subjects there are no standard outcome measures, and often only motor disability is used without any objective and volitional test. In particular, respiratory function tests, and cardiorespiratory disability assessment at rest and during exercise are often underused [9]. No study has described a complete, objective and volitional rehabilitative evaluation of general and respiratory disability; instead, in this field subjective measures such as questionnaires or scales are often used per se [9]. The execution of volitional tests is often difficult in this population as such tests require strong patient participation. However, volitional tests may represent an added value for the rehabilitative setting in that they offer information about specific single functions or activities (e.g., walking ability, ability to reach/maintain upright position, functional use of upper limbs, etc.) and allow a greater estimate of the risk of complications and more information for selecting the most appropriate rehabilitation program. For this reason, we considered several voli-
Table 4. Univariate regression model of Dyspnea Perception (Borg Score) as dependent variable: relationship with other covariates (volitional tests) at discharge.

\begin{tabular}{lcccc} 
Models & Slope & $\mathrm{t}$ & $\begin{array}{c}\text { P-value } \\
\text { coefficient }\end{array}$ & $\mathrm{R}^{2}$ \\
FEV 1 \% pred & -0.036 & -2.87 & 0.009 & 0.273 \\
\hline FVC \% pred & -0.036 & -2.33 & 0.029 & 0.198 \\
\hline MIP \% pred & -0.038 & -2.02 & 0.052 & 0.113 \\
\hline MEP \% pred & -0.0216 & -1.69 & 0.101 & 0.070 \\
\hline PCEF, L/min & -0.012 & -3.59 & 0.002 & 0.381 \\
\hline QMS score & 0.023 & 0.09 & 0.926 & 0.0002 \\
\hline BMS score & -0.01 & -0.04 & 0.968 & 0.0000 \\
\hline DMS score & -0.0009 & -0.04 & 0.971 & 0.0000 \\
\hline Sit-to-stand, n of repetitions & -0.04 & -0.81 & 0.424 & 0.010 \\
\hline Takahashi, sec & -0.005 & -1.60 & 0.116 & 0.050 \\
\hline 6MWT, meters & -0.006 & -2.16 & 0.036 & 0.082 \\
\hline
\end{tabular}

MIP: maximal inspiratory pressure; MEP: maximal expiratory pressure; PCEF: peak cough expiratory flow; QMS: quadriceps muscle strength; BMS: biceps muscle strength; DMS: latissimus dorsi and teres major muscle strength; 6MWT: six-minute walking test.

tional tests, commonly used as outcomes of a rehabilitative program, in a unique triage package. These tests may be used to guide global rehabilitative assessment and needs in post-critically ill subjects.

Spirometry is the most important measure for determining severity of airway obstruction (FEV1) and lung function (FVC) [28]. The FEV1 and FVC values are currently used in the diagnosis, staging and treatment of COPD, both being measurements that are highly reproducible [12]. MIP and MEP are indirect measures of respiratory muscle strength [13], while PCEF reflects the patient's ability to protect airways and remove secretions [15]. Peripheral muscle function was tested by manual muscle testing [MMT)] using the MRC scale [18] and describing one leading muscle for the upper limb, lower limb and trunk districts. Although the debate is open about the best methodology to use, MMT is currently the preferred diagnostic tool for evaluating the presence of ICU-acquired weakness [9] has proven reliability [29], and shows a high inter-observer agreement [30]. The improvement in effort tolerance is one of the main goals of pulmonary rehabilitation also in subjects who have survived a long stay in the ICU [31]. The sit-to-stand test is less stressful, less likely 
to cause hemodynamic variation, easier to apply and more informative than the 6MWT [32,33]. The Takahashi Test is an unsupported incremental upper limb exercise test that reflects the arm use during activities of daily living: it is validated and reproducible [21]. The 6MWT is the most frequently used field test: it is validated and standardized, it is relatively simple to perform, well tolerated and reflects everyday activity [34], and it correlates with lung function, health status, and maximal $\mathrm{VO}_{2}$ [34].

All the proposed measures are based on the assumption that subjects can collaborate actively in performing the maneuver. We maintain that many critically ill subjects are not able to perform one or more of these tests in the first few days of rehabilitation in a hospital facility, while they may be able to do so at the time of discharge. We also believe that the inability per se to perform volitional tests is an important clinical indicator and can be used as a measure of outcome, to assess the individual's rehabilitation needs and to allocate subjects to different interventions or rehabilitation facilities.

As expected, i) at baseline only a small number of subjects was able to perform respiratory function and effort tolerance tests, whereas a larger number of subjects had sufficient muscular activity to be able to perform dedicated tests in selected peripheral muscles at the same time (Figure 1); ii) at discharge, a large number of tests were completed with higher scores obtained; however more than $70 \%$ of subjects were still unable to perform leg effort tolerance tests, while more than $50 \%$ were unable to perform the arm effort tolerance tests demonstrating the objective high residual disability of this category of subjects; iii) at discharge there was a significant difference in ability to perform all tests between cardiorespiratory and neurological patients. This fact implies that each individual patient may have a different response to hospital rehabilitation intervention, and possibly requires a different approach or more time to reach a specific goal in each domain.

The relationship between peripheral muscle measures and Barthel Index, as well as between respiratory functional tests and dyspnea don't surprise us and supports the great necessity of a global assessment.

\section{Clinical implications}

The application of volitional tests - evaluating outcomes commonly used in a pulmonary rehabilitation program - is of clinical importance because allows to face up to different aspects of the physical and respiratory disability. However, the feasibility of those tests in the P-ICU setting, both at admission and discharge, in general has been shown to be low.

For these reasons, the package of evaluation tests administered during the pulmonary rehabilitation program in P-ICU should be tailored to the single patient, identifying his/her specific abilities and needs for rehabilitation (i.e., of the upper limbs, lower limbs, or respiratory muscles), being a global assessment not viable. The introduction of new outcome measures including non-volitional tests (i.e., cross sectional muscular area, p-twitch of diaphragm muscle, etc.) that may be administered to everyone should also be argued.

\section{Limitations}

The main limitation of this study concerns its observational nature. No specific test to diagnose "ICU-acquired" weakness was conducted.

\section{Conclusions}

For the first time, multiple volitional rehabilitation evaluations were carried out in P-ICU subjects undergoing a rehabilitation program to determine which type of physical rehabilitation intervention was feasible for post-critical illness patients. At admission, peripheral muscle evaluations were proved to be the most feasible volitional tests, while spirometry and leg effort tolerance were the least feasible. The performability of the tests increased over time and, at discharge, the motor disability was mainly related to peripheral muscle strength. Specific improvement (i.e., increase in $6 \mathrm{MWT}$ ) was found only in some patients suggesting that the tests should be tailored patient by patient to reach the more proper rehabilitative outcome.

This low viability describes the need of new objective outcome measures (also non-volitional) that might be administered in patients that are not able to perform some of them at the beginning of rehabilitation. Future studies should investigate whether the evaluation of these patients might be linked to a protocol-driven intervention with the aim to produce better rehabilitation outcomes in this specific population.

\section{References}

1. Vitacca M, Nava S. Incomplete network for survivors of catastrophic illness after release from ICUs. Respir Care 2013;58:383-5.

2. Topp R, Ditmyer M, King K, et al. The effect of bed rest and potential of rehabilitation on subjects in the Intensive care unit. AACN Clin Issues 2002;13:263-76.

3. Nava S, Vitacca M. Chronic ventilator facilities. In: Tobin MJ, ed. Principles and practice of mechanical ventilation, 3rd ed. New York: McGraw-Hill; 2013: pp. 777-92.

4. Corrado A, Roussos C, Ambrosino N, et al. European respiratory society task force on epidemiology of respiratory intermediate care in Europe. Respiratory intermediate care units: a European survey. Eur Respir J 2002;20:1343-50.

5. Bailey P, Thomsen GE, Spuhler VJ, et al. Early activity is feasible and safe in respiratory failure subjects. Crit Care Med 2007;35:139-45.

6. Thomsen GE, Snow GL, Rodriguez L, Hopkins R0. Subjects with respiratory failure increase ambulation after transfer to an intensive care unit where early activity is a priority. Crit Care Med 2008;36:1119-24.

7. Clini EM, Crisafulli E, Antoni FD, et al. Functional recovery following physical training in tracheotomized and chronically ventilated subjects. Respir Care 2011;56:306-13.

8. Vitacca M, Paneroni M, Peroni R, et al. Effects of a multidisciplinary care program on disability, autonomy and nursing needs in subjects recovering from acute respiratory failure in a chronic ventilatory facility. Respir Care 2014;59:1863-71.

9. Elliott D, Denehy L, Berney S, Alison JA. Assessing physical function and activity for survivors of a critical illness: A review of instruments. Aust Crit Care 2011;24:155-66.

10. Connolly B, O’Neill B, Salisbury L, Blackwood B; Enhanced Recovery After Critical Illness Programme Group. Physical rehabilitation interventions for adult patients during critical illness: an overview of systematic reviews. Thorax 2016;pii: thoraxjnl-2015-208273.

11. Knaus WA, Draper EA, Wagner DP, Zimmerman JE. Acute physiology and chronic health evaluation II (APACHE II): a severity of disease classification system. Crit Care Med 1985;13:818-29.

12. Miller MR, Hankinson J, Brusasco V, et al. ATS/ERS Task Force. Standardisation of spirometry. Eur Respir J 2005;26:319-38.

13. Black LF, Hyatt RE. Maximal respiratory pressures: normal values and relationship to age and sex. Am Rev Respir Dis 1969;99:696-702.

14. Evans JA, Whitelaw WA. The assessment of maximal respiratory mouth pressures in adults. ATS/ERS Statement on Respiratory Muscle Testing. Respir Care 2009;54:1348-59.

15. Leiner GC, Abramowitz S, Small MJ, Stenby VB. Cough peak flow. Am J Med Sci 1966;251:211-4.

16. Sancho J, Servera E, Diaz J, Marín J. Comparison of peak cough flows measured by pneumotachograph and a portable peak flow meter. Am J Phys Med Rehabil 2004;83:608-12. 
17. Kendall FP, Kendall-McCreary E, Provance P. Muscles: Testing and Function. Fourth Edition. In: Williams \& Williams, editors. London: Lippincott; 1993.

18. Hough CL, Lieu BK, Caldwell ES. Manual muscle strength testing of critically ill subjects: Feasibility and interobserver agreement. Crit Care 2011;15 R43.

19. Clarkson HL, Gilewich GB. Muscoloskeletal assessment: joint range of motion and manual muscle strength. 2nd ed. Baltimore, USA; Williams \& Wilkins; 1989.

20. Jones CJ, Rikli RE, Beam WC. A 30-s chair-stand test as a measure of lower body strength in community-residing older adults. Res Q Exerc Sport 1999;70:113-9.

21. Takahashi T, Jenkins SC, Straussm GR, et al. A new unsupported upper limb exercise test for subjects with chronic obstructive pulmonary disease. J Cardiopulm Rehabil 2003;23:430-7.

22. ATS Committee on Proficiency Standards for Clinical Pulmonary Function Laboratories ATS statement: guidelines for the six-minute walk test. Am J Respir Crit Care Med 2002;166:111-7.

23. Braakman R, Avezaat CJ, Maas AI, et al. Inter observer agreement in the assessment of the motor response of the Glasgow 'coma' scale. Clin Neurol Neurosurg 1977;80:100-6.

24. Quanjer PH, Lebowitz MD, Gregg I, et al. Peak expiratory flow: conclusions and recommendations of a Working Party of the European Respiratory Society. Eur Respir J 1997;24:2S-8.

25. Mahoney FI, Barthel DW. Functional evaluation: the Barthel Index. A simple index of independence useful in scoring improvement in the rehabilitation of the chronically ill. Md State Med J 1965;14:61-6.
26. Borg G. Psychophysical bases of perceived exertion. Med Sci Sports Exerc 1982;14:377-81.

27. Herridge MS, Tansey CM, Matté A, et al. Canadian Critical Care Trials Group: Functional Disability 5 Years after Acute Respiratory Distress Syndrome. N Engl J Med 2011;364:1293-304.

28. Global Initiative for Chronic Obstructive Lung Disease. Global strategy for the diagnosis, management, and prevention of COPD 2011. Accessed on: 29 June 2016. Available from: http://goldcopd.org

29. Vanpee G, Hermans G, Segers J, Gosselink R. Assessment of Limb Muscle Strength in critically ill subjects: A systemic review. Crit Care Med 2014;42:701-11.

30. Hermans G, Clerckx B, Vanhullebusch T, et al. Interobserver agreement of medical research council sum-score and handgrip strength in the intensive care unit. Muscle Nerve 2012;45:18-25.

31. Calvo-Ayala E, Khan BA, Farber M0, et al. Interventions to improve the physical function of ICU survivors: a systematic review. Chest 2013;144:1469-80.

32. Ozalevli S, Ozden A, Itil 0, Akkoclu A. Comparison of the Sit-toStand Test with 6 min walk test in subjects with chronic obstructive pulmonary disease. Respir Med 2007;101:286-93.

33. Spruit MA, Watkins ML, Edwards LD, et al. Evaluation of COPD Longitudinally to Identify Predictive Surrogate Endpoints (ECLIPSE) study investigators. Determinants of poor 6-min walking distance in subjects with COPD: the ECLIPSE cohort. Respir Med 2010;104:849-57.

34. Brown CD, Wise RA. Field tests of exercise in COPD. The six-minute walk test and the shuttle walk test. COPD 2007;4:217-23. 